Measur ement of a magnet i c i sl and wi th a tangent $i$ al I y vi ewi ng fast-framing CCD camer a i $n$ the Large Hel i cal Devi ce

\begin{tabular}{|l|l|}
\hline $\begin{array}{l}\text { jour nal or } \\
\text { publ i cat i on } \mathrm{ti} \text { t l e }\end{array}$ & I EEE Tr ansact i ons on PI asma Sci ence \\
\hline vol une & 30 \\
\hline number & 1 \\
\hline page $\mathrm{r}$ ange & $82-83$ \\
\hline year & $2002-08$ 07 \\
\hline URL & ht t p: //hdl . handl e. net /10655/00012855 \\
\hline
\end{tabular}




\title{
Measurement of a Magnetic Island with a Tangentially Viewing Fast- framing CCD Camera in the Large Helical Device
}

\author{
M. Shoji, K. Yamazaki, A. Komori and H. Yamada
}

(a) Measurement

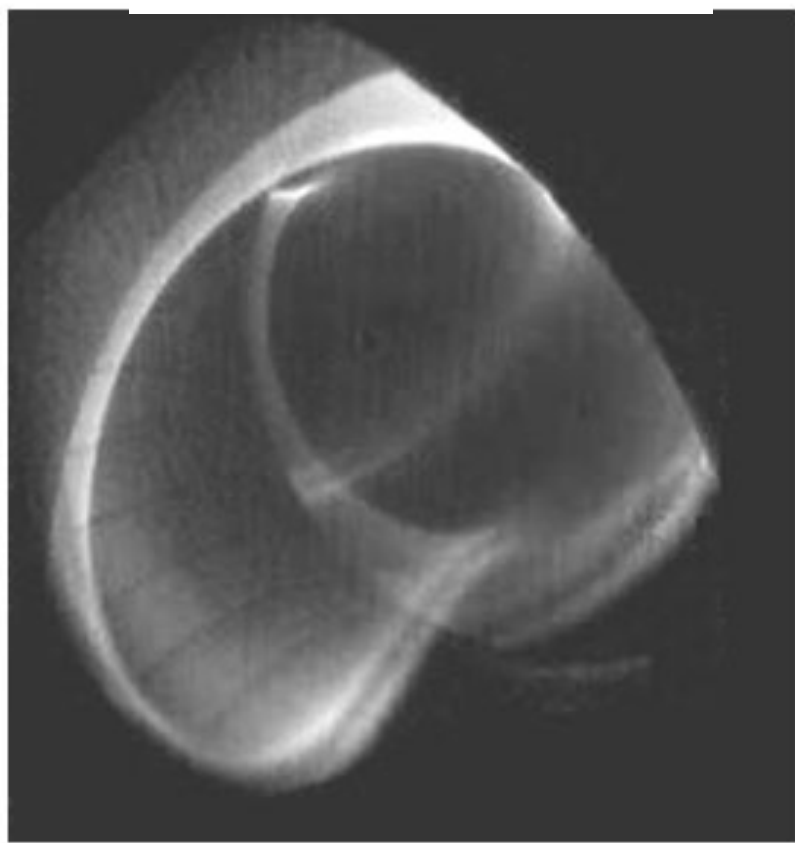

(b) Calculation

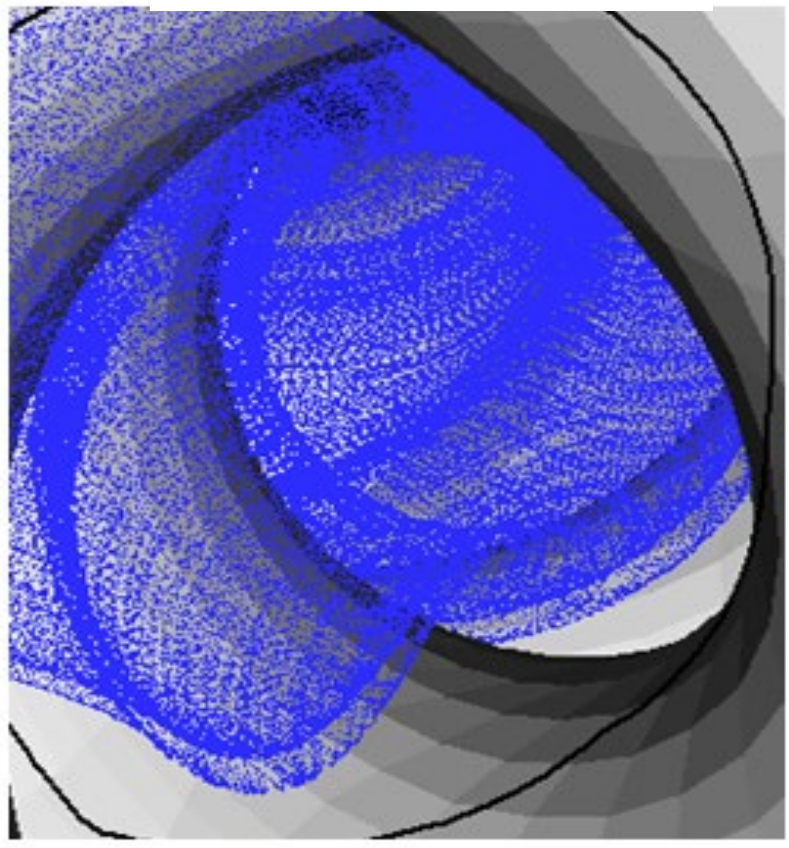

Fig. 1. (a) A measured line emission image from carbon ions (CIII) taken by the tangentially viewing fast-framing CCD camera during the termination phase of the plasma discharge, showing two stripes in the plasma periphery; (b) An image of calculated three-dimensional magnetic field lines on the edge of the $m=1$ magnetic island (blue dots) viewed from the tangential port. The right side and left side in these figures correspond to the outer and inner direction on the major radius, respectively.

\begin{abstract}
Reduction of magnetic islands induced by an error field is essential to achieving high-performance plasmas in helical/stellarator systems. Magnetic islands were observed in the Large Helical Device by applying a fluorescent technique with an electron gun and a fluorescent mesh. Two stripes of impurity line emission profiles were observed with a tangentially viewing fastframing CCD camera during the termination phase of the plasma discharge. A three-dimensional calculation of magnetic field lines on the magnetic island shows that the two stripes correspond to the magnetic islands. The images of the impurity line emission can contribute to identifying magnetic islands and the investigation of the physical mechanisms of plasma confinement in the island.
\end{abstract}

Index Terms - Fast-framing CCD camera, magnetic island, impurity line emission, large helical device (LHD).

The Large Helical Device (LHD) is a helical plasma confinement machine with the following design values: a plasma major radius of $3.90 \mathrm{~m}$, and an averaged plasma radius of $0.65 \mathrm{~m}$ [1]. The plasma confining magnetic field is produced by super-conducting coils which consist of two twisted helical coils $(l=2, m=10)$, and three pairs of poloidal coils. Here, $l$ and $m$ refer to the poloidal and the toroidal periodicity of the helical magnetic field, respectively. The designed rotational transform on the magnetic axis and the last closed magnetic surface are 0.4 and 1.2 in the standard magnetic configuration. Hence, a major low-order rational surface $m / n=1 / 1$ exists in the plasma confinement region, where $m$ and $n$ are the poloidal and the toroidal mode numbers, respectively. An $m=1$ magnetic island can be formed on the rational surface by an effect with an error magnetic field induced by ferromagnetic objects or by an 
alignment error of the coils. The width of the magnetic island $\left(W_{m n}\right)$ can be approximated by

$$
W_{m n} \approx 4 \sqrt{2 \pi R B_{r m n} /\left(m B_{t} d \imath / d r\right)},
$$

where $R$ is the averaged minor radius where the magnetic island is formed, $B_{r m n}$ is the radial Fourier component of the error magnetic field, $B_{t}$ is the toroidal magnetic field, and $t$ is the rotational transform.

Magnetic surfaces are basic reference parameters for analyzing plasma confinement. Therefore, we experimentally measured magnetic surfaces with high magnetic fields $(\sim 3 T)$ by applying a fluorescence technique using an electron gun, a fluorescent mesh and a Peltier cooled CCD (Charge-Coupled Device) camera. An $m=1$ magnetic island was observed inside the last closed flux surface, which indicates the presence of an error field in the plasma confinement region.

In helical/stellarator plasma confinement systems, reduction of magnetic islands is an important issue for achieving high-performance plasmas. We can flexibly control the width and poloidal position of the magnetic island by changing the current configuration of the LID (Local Island Diverter) coils. The coils are twenty toroidally distributed magnetic coils installed on and bellow an LHD cryostat so as to form $n / m=1 / 1,1 / 2$ radial Fourier components of the magnetic fields. We have succeeded in minimizing the island by optimizing the configuration of the coil currents, thereby enabling storage in the plasma of large energies $\left(W_{p} \sim 1 \mathrm{MJ}\right)$.

For monitoring the LHD plasma and plasma-wall interactions, we have used a tangentially viewing fastframing CCD camera which measures impurity line emission profiles in the plasma periphery without image intensifiers. The CCD camera (KODAK Motion Corder Analyzer SR$1000, f=1.4$ ) has a maximum frame rate of 1000 frames per second and a maximum image resolution of $512 \times 480$ pixels. The data of images were stored in RAM memory in the camera controller. The data were converted to the video signal (30 frames per second) after a plasma discharge, and transferred to video tape recorder. An interference filter which transmits line emission from carbon ions (CIII: $\lambda=465.8 \mathrm{~nm}$ ) was mounted on the front of the camera. The CIII line is found to be a prominent line in the LHD plasma because of carbon diverter plates installed along strike points on the vacuum vessel. We observed the discrete transition of the line emission profile from radially outside to inside of the plasma during the termination phase of the plasma discharge, and acquired the image of two stripes of the line emission at the time when the electron temperature around the island is low (in the range of several tenth electron volts). This is because the CIII line emission only occurs in low electron temperature. Figure 1 (a) is a measured line emission image (exposure time $\Delta t=4 \mathrm{~ms}$ ) for $B_{t}=2.83 \mathrm{Tesla}$, the position of the magnetic axis $R_{0}=3.5 \mathrm{~m}$ without the LID coil currents.

The radial positions of the stripes are likely to agree with that of the $m=1$ magnetic island observed in the magnetic surface measurement. To confirm the correspondence, we calculated the three-dimensional structure of the magnetic field lines on the island while including the effect of the error field. Figure 1 (b) shows the image of the calculated magnetic field lines (blue dots) viewed from the tangential port, which corresponds well to the measurement. The calculated images agree with the measurements for various configurations of the LID coil currents. No observable two stripes appeared in optimized LID configuration for minimizing the island. These results imply that we can easily identify magnetic islands by imaging line emission without conventional time-consuming measurement of the magnetic surfaces.

The two stripes of the emission along the magnetic island suggest that the electron temperature at the edge of the island is significantly lower than that inside the island. This can be caused by energy confinement effect in the island. A detailed analysis of the time evolution of the line emission may be helpful to investigating plasma confinement properties and impurity transport physics in magnetic islands.

\section{REFERENCES}

[1] A. Iiyoshi, A. Komori, A. Ejiri, M. Emoto, H. Funaba, M. Goto, K. Ida, H. Idei, S. Inagaki, S. Kado, O. Kaneko, K. Kawahata, T. Kobuchi, S. Kubo, R. Kumazawa, S. Masuzaki, T. Minami, J. Miyazawa, T. Morisaki, S. Morita, S. Murakami, S. Muto, T. Mutoh, Y. Nagayama, Y. Nakamura, H. Nakanishi, K. Narihara, K. Nishimura, N. Noda, S. Ohdachi, N. Ohyabu, Y. Oka, M. Osakabe, T. Ozaki, B.J. Peterson, A. Sagara, S. Sakakibara, R. Sakamoto, H. Sasao, M. Sasao, K. Sato, M. Sato, T. Seki, T. Shimozuma, M. Shoji, H. Suzuki, Y. Takeiri, K. Tanaka, K. Toi, T. Tokuzawa, K. Tsumori, K. Tsuzuki, K.Y. Watanabe, T. Watari, H. Yamada, I. Yamada, S. Yamaguchi, M. Yokoyama, R. Akiyama, H. Chikaraishi, K. Haba, S. Hamaguchi, M. Iima, S. Imagawa, N. Inoue, K. Iwamoto, S. Kitagawa, J. Kodaira, Y. Kubota, R. Maekawa, T. Mito, T. Nagasaka, A. Nishimura, C. Takahashi, K. Takahata, Y. Takita, H. Tamura, T. Tsuzuki, S. Yamada, K. Yamaguchi, N. Yanagi, H. Yonezu, Y. Hamada, K. Matsuoka, K. Murai, K. Ohkubo, I. Ohtake, M. Okamoto, S. Satoh, T. Satow, S. Sudo, S. Tanahashi, K. Yamazaki, M. Fujiwara and O. Motojima, "Overview of the Large Helical Device project," Nucl. Fusion, vol. 39, pp. 1245-1256, Sep 1999. 